\title{
Ações de Enfermagem no cuidado à criança na atenção primária durante a pandemia de COVID-19
}

\author{
Nursing actions in child care in primary care during the COVID-19 pandemic \\ Acciones de enfermería en el cuidado infantile en atención primaria durante la pandemia de COVID-19 \\ Beatriz Rosana Gonçalves de Oliveira Tos0 ${ }^{1}$ (D) https://orcid.org/0000-0001-7366-077X \\ Cláudia Silveira Viera ${ }^{1}$ (D) https://orcid.org/0000-0002-0900-4660 \\ Maria Cândida Carvalho Furtado² (D) https://orcid.org/0000-0001-7942-4110 \\ Poliana Castro de Resende Bonati ${ }^{3}$ (D) https://orcid.org/0000-0002-7423-4438
}

\section{Resumo}

Objetivo: Verificar a atuação do enfermeiro de atenção primária, em unidades básicas de saúde tradicionais e unidades de saúde da família, nas ações de cuidado de rotina à saúde da criança, durante o período de pandemia da COVID-19.

Métodos: Pesquisa qualiquantitativa realizada em junho e julho de 2020; aplicou-se formulário online para 80 enfermeiros de três municípios brasileiros. Análise descritiva e análise de conteúdo.

Resultados: Identificou-se três categorias analíticas: 0 antigo e 0 atual: desafios para 0 cuidado da criança; Impacto e mudança: ações para continuidade da atenção à criança; Certezas/incertezas: caminhos para o cuidado integral. Uso de aplicativos de mensagens e telefone para atendimento remoto, inclusão de orientações sobre prevenção da COVID-19. Atendimento presencial para crianças de risco, consultas espaçadas e medidas preventivas nos ambientes da unidade.

Conclusão: Atendimento presencial mantido, independentemente do tipo de unidade, principalmente as crianças em situações de vulnerabilidade, sendo incluídas novas ferramentas tecnológicas para o cuidado em atendimento remoto.

\section{Abstract}

Objective: To verify the actions of primary care nurses, in traditional basic health units and family health units, in routine childcare actions during the COVID-19 pandemic period.

Methods: Qualitative and quantitative research conducted in June and July 2020; an online form was applied to 80 nurses from three Brazilian municipalities. Descriptive analysis and content analysis.

Results: Three analytical categories were identified: The old and the current: challenges for childcare; Impact and change: actions to continue childcare; Certainty / uncertainty: paths to comprehensive care. Use of messaging and telephone applications for remote assistance, inclusion of COVID-19 prevention guidelines. Face-to-face care for children at risk, spaced consultations, and preventive measures in the unit's environments.

Conclusion: Face-to-face service maintained, regardless of the type of unit, mainly to child in situations of vulnerability, including new technological tools to care in remote services modality.

\section{Resumen}

Objetivo: Verificar las acciones de las enfermeras de atención primaria, en las unidades básicas de salud tradicionales y las unidades de salud familiar, en las acciones de cuidado infantil de rutina durante el período de la pandemia de COVID-19.

Métodos: Investigación cualitativa y cuantitativa realizada en junio y julio de 2020; Se aplicó un formulario en línea a 80 enfermeras de tres municipios brasileños. Análisis descriptivo y análisis de contenido.

Resultados: Se identificaron tres categorías analíticas: la antigua y la actual: desafíos para el cuidado infantil; Impacto y cambio: acciones para continuar el cuidado infantil; Certeza / incertidumbre: caminos hacia la atención integral. Uso de aplicaciones de mensajería y teléfono para asistencia remota, inclusión de pautas de prevención COVID-19. Atención cara a cara para niños en riesgo, consultas espaciadas y medidas preventivas en los entornos de la unidad.

Conclusión: Atendimento presencial mantido, independientemente del tipo de unidade, principalmente como crianças em situacionesções de vulnerabilidade, sendo incluídas novas ferramentas tecnológicas para o cuidado em atendimento remoto.

\section{Como citar:}

Toso BR, Vieira CS, Furtado MC, Bonati PC. Ações de Enfermagem no cuidado à criança na atenção primária durante a pandemia de COVID-19. Rev Soc Bras Enferm Ped. 2020;20(Especial COVID-19):6-15.

\section{Descritores}

Enfermagem pediátrica; Atenção primária à saúde; Saúde da criança; Cuidado de enfermagem: Coronavirus; COVID-19

\section{Keywords}

Pediatric nursing; Primary health care; Child health; Nursing care; Coronavirus; COVID-19

\section{Descriptores}

Enfermería pediátrica; Atención primaria de salud; Salud de los niños; Cuidado de enfermería; Coronavirus; COVID-19 


\section{Introdução}

A Política Nacional de Atenção Integral à Criança (PNAISC), normatizada pela portaria $\mathrm{n}^{\mathrm{o}} 1.130$, de 5 de agosto de 2015,(1) traz entre seus objetivos o acompanhamento do crescimento e desenvolvimento saudável das crianças, procurando otimizar ações dos serviços públicos de saúde no sentido de proteger e promover a saúde da criança, além de prevenir seu adoecimento. Essas ações ocorrem atualmente no Brasil, para o seguimento da criança, em sua grande maioria, por meio da Atenção Primária a Saúde (APS).

O acompanhamento da criança na APS tem continuidade no retorno da mãe e do recém-nascido para casa, quando, ainda na primeira semana de vida, a família deve receber uma visita da equipe de saúde da APS, seja Unidade Básica de Saúde (UBS) ou Estratégia Saúde da Família (ESF), para fazer orientações e esclarecer dúvidas no cuidado ao recém-nascido, ${ }^{(2)}$ sendo agendada a primeira consulta de puericultura. A partir deste momento, a criança passará a ser acompanhada pela equipe da APS em no mínimo, seis consultas no primeiro ano de vida, duas no segundo ano de vida, e pelo menos uma consulta de puericultura ao ano, até completar cinco anos. ${ }^{(2)}$

Além disso, outras ações dirigidas a criança em unidades de APS são as imunizações, as consultas médicas, as visitas domiciliares, o encaminhamento para os programas sociais. Desse modo, mediante a importância da atenção a criança para acompanhar seu crescimento e desenvolvimento saudável, na atenção primária, torna-se necessário indagar: a atuação do enfermeiro de atenção primária, nas ações de cuidado à criança, durante o período de pandemia do COVID-19 continuaram a acontecer de forma similar ao executado antes do período de pandemia?

Este questionamento é premente frente a atual situação experenciada pelo mundo e pelos serviços de saúde em decorrência da pandemia desencadeada pela doença do Corona Vírus 2 (SARS-CoV2). A referida doença é caracterizada como uma infecção respiratória aguda potencialmente grave, denominada síndrome respiratória aguda grave Coronavírus 2 (SARS-CoV-2). As taxas de letalidade e mortalidade têm sido variáveis ao redor do mundo, a depender das medidas de contenção de transmissão adotada pelos diferentes países, sendo uma ameaça importante e urgente à saúde global. Desde o surto no início de dezembro de 2019 na província de Hubei, na República Popular da China, o número de pacientes confirmados para a doença ultrapassou 13.286.18917 em todo o mundo, e o número de pessoas infectadas é maior, devido à ausência de testagem para todos os possíveis casos. Mais de 578.610 pessoas morreram de infecção pelo COVID-19, até 15 de julho de 2020. No Brasil, nessa mesma data, tem-se 1.926 .824 casos confirmados e 74.133 óbitos. ${ }^{(3,4)}$

As crianças são tão propensas quanto os adultos a se infectarem com o vírus, mas têm menos sintomas e menos doenças graves. Na população pediátrica infectada foi evidenciado, em relatos de séries de casos, até o momento, que $83 \%$ das crianças adquiriram a infecção de membros da família. Os sintomas mais comuns foram tosse, febre e faringite. Outros sintomas foram congestão nasal, rinorreia, taquipneia, chiado no peito, diarreia, vômito, dor de cabeça e fadiga. Os parâmetros dos testes laboratoriais foram apenas minimamente alterados. Os achados radiológicos eram inespecíficos e incluíam infiltrados unilaterais ou bilaterais com, em alguns casos, opacidades em vidro fosco ou consolidação com um sinal de halo circundante. As crianças raramente precisavam de internação em unidades de terapia intensiva (3\%) e, até o momento, apenas um pequeno número de mortes foi relatado em crianças em todo o mundo. Dentre as complicações neonatais tem-se desconforto respiratório ou pneumonia, coagulação intravascular disseminada e asfixia. ${ }^{(5)}$

Para evitar que o vírus se espalhe em proporções que o Sistema Único de Saúde (SUS) não possa ter condições de dar as respostas esperadas nos três níveis de atenção à saúde, medidas de prevenção primária, já utilizadas em países como Coreia do Sul e Alemanha, foram sugeridas pelo Ministério da Saúde brasileiro, tais como o isolamento social, a quarentena de 14 dias em casos suspeitos, evitar contato com pessoas vulneráveis e vacinar antecipadamente a população para outras viroses respiratórias. Na prevenção secundária, encontram-se medidas como isolar imediatamente casos confirmados com sintomas leves em ambiente domiciliar e notificar os casos suspeitos as autoridades sanitárias. ${ }^{(6)}$

Ao se organizar para atender as demandas de saúde em momento de pandemia, os serviços municipais modificaram o funcionamento das unidades de APS, muitas das quais permaneceram fechadas 
desde março desse ano, ou então dirigiram seu foco de cuidado somente para casos da COVID-19. Nesse sentido, estudos devem ser realizados em tempo real, para que se conheça mais sobre como desenvolver o trabalho rotineiro das unidades de saúde na presença desse novo vírus.

Estima-se que em nossa realidade, com as mudanças ocorridas no processo de trabalho na APS decorrentes da pandemia de COVID-19, possam existir fatores que dificultem a execução da prática de cuidado a saúde às crianças, os quais esperamos identificar. Assim, este estudo torna-se relevante à medida que se propõe a reconhecer fatores que promovem ou dificultam o exercício das ações de cuidado à criança, pois estas atividades podem influenciar no processo de promoção de saúde e prevenção de doenças das mesmas.

Isto posto, concebe-se que as atividades de cuidado, clínicas, dentre elas a consulta de enfermagem em puericultura, as imunizações, as visitas domiciliares (VD), possam estar sendo realizadas de maneira precária ou nem sendo realizadas. Para tanto, o objetivo do estudo foi verificar a atuação do enfermeiro de atenção primária, em unidades básicas de saúde tradicionais e unidades de saúde da família, nas ações de cuidado de rotina à saúde da criança, durante o período de pandemia do COVID-19.

\section{Métodos}

Estudo qualiquantitativo, de natureza aplicada, descritiva, exploratória e transversal, realizado em três municípios de médio porte, sendo um no oeste do estado do Paraná, com população estimada de 316.226 habitantes, um no estado de São Paulo, um com população estimada de 703.293 habitantes e outro em Minas Gerais, na mesorregião do Triângulo Mineiro/Alto Parnaíba, com população estimada, em 2018, em 683.247 habitantes.

O município paranaense conta com 43 unidades, destas 22 são Unidades Básicas de Saúde tradicionais e 21 Unidades de Saúde da Família, distribuídas em três distritos de saúde. O município paulista dispõe de 48 unidades de atenção primária, sendo 32 unidades básicas tradicionais e/ou mistas, centro de saúde e 16 unidades de saúde da família, distribuídas em cinco distritos de saúde. O município mineiro tem as unidades de saúde regionalizadas em quatro setores sanitários e possuem, no total, 81 equipes de saúde da família, distribuídas em 56 Unidades Básicas de Saúde da Família.

A população da pesquisa foi composta por enfermeiros vinculados às unidades de atenção primária dos três municípios. A coleta de dados ocorreu por meio de formulário do google forms, o qual foi enviado por e-mail a todos os enfermeiros da atenção primária dos municípios e aqueles que aceitaram participar tiveram o prazo de uma semana, prorrogável por mais uma, para responder a pesquisa.

O instrumento de coleta de dados incluiu informações de caracterização dos participantes, como idade, sexo, tempo de formação e de atuação na APS, especializações. Também, questões específicas sobre o tema do estudo, como a realização de atendimentos a crianças na unidade de trabalho durante o período da pandemia, e se não, por quais motivos.

A análise dos dados dicotômicos quantitativos foi realizada utilizando-se tratamento de estatística descritiva simples, apresentando-se os resultados em números inteiros e proporcionais. ${ }^{(7)} \mathrm{A}$ análise de dados descritivos ocorreu por meio de análise de conteúdo, seguindo-se os passos: pré-análise, exploração do material, tratamento dos resultados obtidos e interpretação. ${ }^{(8)} \mathrm{Na}$ pré-análise, se organizou os conteúdos em quadros sistematizadores para visualizar, de maneira abrangente, as respostas em conjunto. Na sequência, fez-se a exploração do material, que possibilitou a construção de categorias que configuram a estrutura do fenômeno estudado. Em seguida, interpretou-se os dados a luz da literatura.

Este estudo seguiu os preceitos éticos da resolução no 466/2012 e foi aprovado pelo Comitê de Ética em Pesquisa com Seres Humanos da Universidade Estadual do Oeste do Paraná, sob CAAE número: 31807214.8.0000.0107 e parecer $\mathrm{n}^{\mathrm{o}}$ 4.072.796. Todos os participantes da pesquisa assinaram o termo de consentimento livre esclarecido.

\section{Resultados}

Atenderem ao convite para participar do estudo 80 enfermeiros dentre o total de 144 unidades; 52 deles indicaram local de trabalho, sendo 41 (78,9\%) de unidades de saúde da família e $11(21,1 \%)$ de unidades com modelo tradicional de atenção à saúde. A média de idade foi de 
37,6 anos; maioria (73; 91,2\%) sexo feminino. Na tabela 1 , observa-se que a maioria tem mais de 10 anos de formação, e com mais de seis anos de atuação na APS. Da formação complementar, 74 (92,5\%) enfermeiros indicaram ter especialização. Do total de participantes, apenas cinco possuem outra atividade laboral, sendo quatro deles como docente (curso técnico e superior).

As especializações mencionadas foram, principalmente, nas áreas de Saúde Pública/Saúde Coletiva/ Saúde da Família; Enfermagem do Trabalho/Saúde do Trabalhador; Gestão em Saúde/ Gestão em Saúde Pública/ Enfermagem em Saúde da Mulher/ Criança/ Idoso; Urgência/Emergência.

Tabela 1. Caracterização dos enfermeiros, segundo formação e trabalho nos três municípios brasileiros

\begin{tabular}{lc}
\hline Variável & $\mathrm{n}(\%)$ \\
\hline Tempo de formação (anos) & \\
1 a 5 & $6(7,5)$ \\
6 a 10 & $24(30)$ \\
10 ou mais & $50(62,5)$ \\
Tempo que trabalha na APS (anos) & \\
$\quad$ Menos de 1 & $6(7,5)$ \\
1 a 5 & $26(32,5)$ \\
6 a 10 & $24(30,0)$ \\
10 ou mais & $24(30,0)$ \\
Formação Complementar (N= 74) & \\
Especialização & $62(83,8)$ \\
Residência em Enfermagem ou Multiprofissional & $5(6,8)$ \\
Mestrado & $12(16,2)$ \\
Doutorado & $1(1,4)$ \\
Outras & $4(5,4)$ \\
Trabalha em outro emprego & \\
Não & $75(93,8)$ \\
Sim & $5(6,2)$ \\
\hline
\end{tabular}

As questões que versaram sobre os atendimentos de saúde nesse momento de pandemia, indicaram alteração na rotina de trabalho e de cuidados voltados à população. Dos participantes, 57 (71,3\%) revelaram que não está acontecendo atendimento para todas as condições de saúde em suas unidades. Os atendimentos de rotina foram adiados e um dos motivos refere-se ao risco de aglomeração (tal alteração diminuiu o fluxo de usuários na unidade).

Os enfermeiros informaram que as unidades de saúde mantiveram rotina de atendimento para mulheres - gestantes e puérperas e para crianças - puericultura ou aquelas caracterizadas como de alto risco (com 11 relatos cada). Também para casos crônicos de alto risco (quatro relatos), queixa aguda (seis relatos) e ainda urgência e emergência (cinco relatos). Contudo, algumas unidades não estão atendendo crianças com risco habitual. Usuários que comparecem às unidades de saúde por demanda espontânea estão sendo triados e devidamente orientados. Quase todos os enfermeiros indicaram fazer o uso de telefone, telemedicina/ teleatendimento como forma de continuar o acompanhamento da população de sua área de abrangência.

Ao serem questionados sobre os atendimentos de crianças desenvolvidos pela APS nesse período da pandemia da COVID 19, todos os enfermeiros apontaram as atividades que permanecem disponibilizadas para essa clientela, conforme tabela 2.

Tabela 2. Ações de cuidado do enfermeiro para crianças em unidades de saúde de três municípios brasileiros, durante pandemia da COVID-19

\begin{tabular}{lc}
\hline Ações de cuidado & $\mathrm{n}(\%)$ \\
\hline Consulta de Enfermagem em Puericultura & $70(87,5)$ \\
Imunizações & $76(95,0)$ \\
Visitas Domiciliares & $53(66,5)$ \\
Visita da primeira semana de vida integral & $42(52,5)$ \\
Busca ativa de faltosos à Imunização & $70(87,5)$ \\
Triagem neonatal biológica (teste do pezinho) & $78(97,5)$ \\
\hline
\end{tabular}

Do total de participantes, 24 enfermeiros indicaram os motivos de não realização de determinadas ações de cuidado para a criança em suas unidades de saúde, como, a necessidade de manutenção do distanciamento social e prevenção do risco de contágio da COVID-19; suspensão de atendimentos de rotina; priorização de casos, acolhimento e consulta somente para queixas agudas.

$\mathrm{Na}$ análise das falas, os conteúdos foram organizados e da exploração do material, emergiram as categorias: $\mathrm{O}$ antigo e o atual: desafios para o cuidado da criança; Impacto e mudanças: ações para continuidade da atenção à criança; Certezas/incertezas: caminhos para o cuidado integral.

\section{0 antigo e 0 atual: desafios para 0 cuidado da criança}

Quando questionados se a unidade de saúde que trabalham está fechada ou mudou a rotina de funcionamento por causa da pandemia, 77 enfermeiros responderam, sendo que 63 enfermeiros (81,3\%) apontaram que continuam realizando orientações às famílias sobre o cuidado às crianças. E as estratégias que têm 
utilizado para contato com os usuários referem-se ao uso do telefone; emprego da teleconsulta/teleatendimento e utilização de aplicativo de mensagens. Ain$\mathrm{da}$, as ferramentas tradicionais foram mantidas, como busca ativa, VD realizadas por médico, ou enfermeiro, ou agente comunitário de saúde e espaçamento de atendimentos presencias das crianças, como forma de evitar aglomerações na unidade de saúde:

Através de teleconsultas médicas e de enfermagem, visitas domiciliares médica, de enfermagem e do agente comunitário de saúde e consultas presenciais para menores de 1 ano com horários espaçados para evitar aglomerações.

Está sendo realizado contato telefônico e via WhatsApp com as orientações referentes a fase do desenvolvimento, utilizadas perguntas chave sobre o estado de saúde e marcos do desenvolvimento, orientação sobre alimentação para faixa etária, prevenção de acidentes, vacinação, sinais e sintomas do COVID e onde procurar atendimento.

Orientações sobre cuidados domiciliares de modo geral; supervisão de sinais de alerta sobre agravos da doença COVID 19 e outros problemas de saúde agudizados.

Estamos atendendo normalmente os casos de puericultura, porém os demais estamos atendendo algumas especificidades em teleconsultas e mesmo presenciais, ex. renovações de receitas, casos crônicos descompensados, alguns encaminhamentos de maior necessidade, alguns casos de vulnerabilidade, os casos são analisados de maneira individual conforme solicitado e identificado pela família, pelos profissionais da unidade ou quando encaminhados por outros órgãos.

Somente estão sendo atendidas presencialmente as crianças de alto risco. As demais estão recebendo orientações via telefone ou WhatsApp, visitas das ACS (Agentes Comunitários de Saúde) e orientações quando vem até a unidade para vacinas.

Busca ativa aos faltosos, vacinas atrasadas, através de visitas domiciliar por Agente Comunitário, envio de cartas, contato telefônico. Orientação quanto uso de máscara e a importância do isolamento social. Orientamos também quanto à importância do agendamento destas crianças nas consultas, não abandonando, assim, o atendimento à criança.

A unidade mudou toda a rotina de funcionamento, não só com crianças. Alguns contatos são realizados por telefone, como as DNV (Declaração de Nascidos Vivos), e orientamos alguns cuidados com o RN (Recém-Nascido) que seria passado durante a visita domiciliar, não receber visitas e evitar saídas desnecessárias entraram para as orientações. $\mathrm{Na}$ primeira consulta (agendada) do $R N$ é permitido somente 1 acompanhante. As vacinas são agendadas para evitar que a criança fique em meio a aglomerações.

Teleconsulta de enfermagem para casos de risco habitual, alto risco e risco intermediário, teleconsulta seguida de consulta de seguimento na unidade com agendamento prévio. Vacinação de rotina com agendamento via telefone.

Estão sendo realizadas busca ativa de vacinas por telefone e WhatsApp, as vacinas estão sendo agendadas com intervalo de horário, as visitas domiciliares estão sendo realizadas com cuidados para prevenção de COVID, com limpeza e desinfecção de todo material e com EPI adequado.

As ações diversificam-se e o que se percebe é a busca constante pela manutenção do atendimento à saúde da criança, quer seja pela presença do profissional no domicílio, quer seja pelo contato remoto. Ademais, a discussão de casos oriundos da VD contribui para que a equipe da unidade de saúde (UBS ou USF) tenha informações sobre a saúde da criança e da família nessa situação de pandemia. As orientações contemplam os cuidados de crescimento e desenvolvimento da criança, como também aqueles referentes à sinais e sintomas da COVID-19.

\section{Impacto e mudanças: ações para continuidade da atenção à criança}

As mudanças ocorridas devido à pandemia foram amplamente consideradas nesse estudo. Para 91,3\% 
dos enfermeiros, as crianças de sua área de abrangência que precisaram de atendimento na unidade de saúde não deixaram de consegui-lo devido as mudanças no atendimento por causa da pandemia. Para 82,0 \% deles, a unidade de saúde tem conseguido acompanhar as crianças menores de dois anos desde o início da pandemia. Ainda, 78,8\% deles acreditam que nenhuma criança que necessitou de referência para outro serviço de saúde, não a conseguiu, por causa ou em decorrência da pandemia. O que se observa, então, é que as crianças têm tido atenção em saúde nesse contexto de pandemia, na ótica dos enfermeiros.

Para aqueles enfermeiros que revelaram mudanças na rotina de cuidado da criança em sua unidade de saúde, solicitou-se indicação de algum modo de continuar a ofertar cuidado seguro e adequado a elas. Grande parte dos participantes considera o atendimento remoto como forma de acompanhar a saúde da criança. Também o aumento gradual de consultas presenciais, que no início da pandemia ficou restrito a determinada faixa etária.

Houve alteração; porém, demos continuidade ao atendimento. Inclusive pelo WhatsApp da unidade, para tirar dúvidas dos pais, ver resultado de exames e orientações gerais.

A unidade está vivendo neste momento várias mudanças nas rotinas. As famílias estão mais apreensivas e resistentes ao acompanhamento na unidade de saúde. Os profissionais estão experimentando novas formas de atendimentos e com isso tentando não reduzir a qualidade dos serviços prestados.

Contatos pelo telefone, WhatsApp e trazer a criança em casos de necessidade para ser avaliada

Teleconsulta e horários exclusivos para atendimentos às crianças.

No início da pandemia, as crianças maiores de 6 meses ficaram sem atendimento; depois aumentamos a faixa para um ano; depois para 2 anos e agora todas as faixas etárias estão sendo atendidas. Para as crianças menores de 1 ano que não compareceram foi feito contato telefônico.
Pessoalmente em alto risco e por teleconsulta e telefone em caso de baixo risco.

Talvez aumentar a higienização do ambiente entre os atendimentos e de forma mais rigorosa, trocar os EPIs (Equipamento de Proteção Individual) utilizados nos atendimentos para evitar infecção cruzada.

\section{Certezas/incertezas: caminhos para o cuidado integral da criança}

Os enfermeiros pontuam adaptação necessária a um momento em que profissionais e famílias/crianças necessitam olhar para aspectos de segurança na prevenção da contaminação pelo vírus, sem descuidar da atenção à saúde infantil.

Sentimentos de medo, insegurança permeiam o cuidado nas unidades de saúde, seja para profissionais ou para usuários. Contudo, as falas caminham para um enfrentamento positivo, na medida em que os profissionais de saúde se apoiam na atenção precisa aos usuários e, com um novo modo de atender. Destaque para prevenção de exposição desnecessária da criança e família e percepção de que cuidados à determinadas crianças compensam o risco de seu comparecimento na unidade de saúde, além da importância da manutenção do vínculo com ela e sua família.

Estamos em um momento de muita incerteza, mas estamos empenhados e motivados a passar todo conforto e acalmar as famílias.

Alterou a rotina e maneira de trabalho. Algumas crianças estão sendo atendidas por teleconsultas. Que era uma modalidade que não utilizávamos.

Cuidado superficial. As famílias estão com medo de ir até a Unidade.

É um momento de adaptação tanto para os profissionais quanto para a população, porém acredito que dentro das possibilidades estamos dando continuidade ao acompanhamento da criança. 
Atendimento a quem procura sob demanda espontânea e com queixas. Acho que devemos ficar atentos para os próximos tempos e redobrar cuidados para com as crianças para não perdermos o vínculo.

Com muita dificuldade visto que o risco de contágio não pode ser menosprezado; porém, o acompanhamento é muito importante.

Momento de ansiedade e apreensão, mas ao mesmo tempo buscando acolher, acompanhar as crianças seja por consulta presencial ou teleconsulta.

A mudança de fluxos a cada 24 horas está causando angústia e dificuldade no manejo para atendimento $e$ encaminhamentos para especialidades, pois muitas [unidades de saúde] pararam o atendimento e sem uma contrarreferência, estamos sem suporte para alguns casos.

Que cada vez mais precisamos reforçar as orientações quanto aos cuidados com a criança no momento da pandemia, pois ainda quando realizamos $V D$ observamos que as crianças estão nas ruas brincando com outras crianças e sem uso de máscaras.

Com muita informação, suporte e atenção às famílias e crianças estamos adaptando todos a esse novo modelo de atendimento e o retorno até então tem sido positivo para família e equipe.

A população está perdendo o vínculo com a Unidade, os acompanhamentos estão se perdendo e voltando para o modelo medicocêntrico e da medicalização.

...as puericulturas de alto risco ... estão sendo realizadas mensalmente ou com maior frequência, de acordo com a necessidade de cada caso, pois o risco benefício justifica estes atendimentos.

Momento delicado. Na minha opinião, os primeiros meses de vida da criança são os mais difíceis $e$ cheios de dúvidas pelas famílias. Talvez essa seja a nossa maior dificuldade. De não poder manter esse contato tão próximo com essas pessoas.

Situações que afetam o cuidado integral:
É uma experiência nova e inimaginável. As dificuldades são: o medo da própria população/famílial paciente com relação a Unidade de Saúde e o risco de contaminação; a falta de compreensão de alguns grupos com relação a realidade atual; os medos da própria equipe de saúde com relação a contaminação o que acaba diminuindo o tempo de atendimento $e$, como consequência, afetando o atendimento integral, pois preconiza-se a criança e acabamos nos esquecendo da família do paciente.

Destaca-se também a importância de pensar para além desse momento de pandemia, de afastamento social e de mudanças nos atendimentos das crianças. Relatos mostram ser preciso monitorar as crianças, orientar os pais/cuidadores sobre o seguimento delas e atentar-se para potenciais riscos futuros para essas crianças, dada ausência/alteração de acompanhamento adequado de seu crescimento e desenvolvimento.

É bastante evidente que muito se perde nesse contexto; porém, ao pesar o risco de trazer esta criança para dentro da unidade neste momento, nos casos de baixo risco e até em alguns de risco intermediário, eu pondero que o teleatendimento é um recurso muito válido. Será necessário ofertar uma atenção especial a todas estas famílias após essa pandemia, porém, no momento, postergar o atendimento presencial é uma medida bastante útil. Por outro lado, caso esse cenário se estenda por muito tempo, o risco de essas crianças de baixo risco e risco habitual, elevarem seus graus de classificação devido à ausência do acompanhamento de indicadores importantes como a medida de perímetro cefálico, o acompanhamento do ganho de peso e do desenvolvimento neuropsicomotor é grande. Assim sendo, eu considero, organizar na unidade um cronograma especial para este público, cumprindo todas as medidas de controle de infecção ambulatorial, para que ao menos 1 avaliação em 3 meses seja realizada.

A equipe deve monitorar suas crianças. Muitas famílias por medo podem ter deixando de leva-las nas consultas de rotina por ter ouvido muitas notícias, por vezes controversas na mídia e no próprio bairro onde a unidade está inserida. O ACS tem um papel de muita importância na rotina da unidade e em tempos de 
pandemia sua função deve ser reorganizada e focada nos casos essenciais como gestante, criança, idosos e outras condições mais vulneráveis.

Apesar de manter os atendimentos para crianças menores de 2 anos, observo que algumas famílias faltaram às consultas agendadas ou não marcaram retorno, algumas por acharem que não estávamos atendendo e outras por medo de trazer seus filhos à unidade e expô-los à doença. Quando identificada falta ou ausência de retornos recentes, tentamos contato por meio telefônico com os pais ou responsáveis para orientar que estamos atendendo e que diversas medidas foram tomadas para garantir maior segurança aos usuários, além de reforçar a importância do seguimento.

Outros percebem manutenção na efetividade do cuidado prestado à criança em seu trabalho cotidiano, com esforços conjuntos da equipe, bem como com apoio da gestão. As falas demonstram que toda forma de contato, presencial ou remoto, deve prevalecer quando se trata da saúde da criança.

Estamos acompanhando crianças de alto e médio risco presencialmente e de baixo risco por contato telefônico.

A atenção à saúde da criança devido a novos protocolos implantados pela SMS não deixa de ser efetiva, pois é realizada busca ativa, orientações por diversas modalidades e em caso de agravamento de casos especiais é mantida também a atenção em saúde da criança.

Apesar de toda parte emocional da equipe ter sido afetada, temos trabalhado para melhorar cada vez mais a atenção à saúde dos usuários da nossa área de abrangência. A gestão tem oferecido suporte e estamos atendendo à distância e presencialmente quando necessário. Acredito que a atenção à saúde da criança está sendo atendida com louvor. Tendo em vista que todas as crianças de 0 a 2 anos estão em acompanhamento.

Está acontecendo de forma tranquila, pois não deixamos de fazer o atendimento dos primeiros dias: puericultura, teste do pezinho e orientações de ama- mentação ao RN. E além de dar suporte via telefone e WhatsApp quando são crianças de médio e baixo risco.

\section{Discussão}

A rotina do trabalho na atenção primária sofreu alterações importantes com a pandemia de COVID-19, conforme retratado pelos enfermeiros do estudo. Usualmente, o enfoque nesse âmbito de atenção é o das ações programadas, para o conjunto dos profissionais, com atendimentos de casos agudos conforme demanda espontânea. ${ }^{(9)}$ Por ora, em decorrência da pandemia, os atendimentos de rotina foram suspensos e/ou adiados em muitas unidades, independente de ser UBS tradicional ou USF, permanecendo abertas àquelas para realizar a triagem dos casos suspeitos da doença.

Especificamente em relação a criança, houve um redirecionamento no enfoque do cuidado, de presencial, em que se priorizaram os casos agudos, para remoto, seja por aplicativos de mensagens, seja por telefone. Rotineiramente, em consultas de enfermagem para puericultura, predominam as orientações sobre o crescimento e desenvolvimento da criança, imunizações, alimentação, higiene, sono e repouso, prevenção de acidentes, entre outras. ${ }^{(10,11)}$

Atualmente, para além dessas orientações mencionadas, os contatos têm sido realizados também para buscar os faltosos nas imunizações e orientar sobre dúvidas a respeito de sinais e sintomas da COVID-19. Essa transformação no cuidado, de presencial a remoto, foi a maior mudança para esse grupo, em especial, mudança essa que teve origem, principalmente, na manutenção do distanciamento social e em medidas de prevenção da contaminação pelo novo Coronavírus, conforme orientam os protocolos sobre a doença. ${ }^{(12)} \mathrm{O}$ emprego das novas tecnologias como as digitais no cotidiano do cuidar na APS, em tempos de pandemia, ajuda a pensar em novas oportunidades de cuidado, como a telemedicina, com potencial para permitir redução de custos e aumento da acessibilidade. ${ }^{(13)}$ Contudo, há que se considerar o preparo da equipe de saúde para que, com prática adequada, seja possível uma implementação eficaz desse recurso.

Para aqueles atendimentos que foram mantidos nas unidades, como as consultas as crianças vulnerá- 
veis e/ou de risco, entre elas as prematuras, e as imunizações na sala de vacinas, houve espaçamento na agenda de consultas, de modo a evitar aglomeração. Além disso, manteve-se a busca ativa dos faltosos nas atividades agendadas, por aplicativo de mensagens e telefone, e ainda por VD, realizada principalmente pelo ACS, mas também por médicos e enfermeiros. Manter essas atividades de monitoramento, que já fazem parte a rotina da atenção primária é importante para preservar o vínculo com as famílias. ${ }^{(14,15)}$

Ademais, destaca-se que nas orientações oferecidas pela equipe de saúde às famílias das crianças, além daquelas próprias para cada idade, foram incluídas as orientações sobre prevenção e sinais e sintomas da COVID-19, em todos os momentos de contato com as famílias, independentemente do meio de comunicação. ${ }^{(6,14)}$ No ambiente físico das unidades, medidas de segurança foram adotadas, como EPI para a COVID-19 acrescidos a rotina da atenção primária, além de as regras de higienização do ambiente se tornarem mais rigorosas, com limpeza dos consultórios entre os atendimentos. ${ }^{(6)}$

Outro aspecto evidenciado nos relatos foi a expressão de sentimentos sobre o momento vivido, na relação família-profissional, experimentado tanto pelos enfermeiros quanto pelas famílias, como medo, insegurança, ansiedade, apreensão, angústia, gerados pela incerteza do momento de pandemia. Tais sentimentos são comuns em momentos de dificuldade e precisam ser considerados no cuidado para evitar sofrimento psíquico ao trabalhador e as famílias. ${ }^{(14,16)}$

Percebeu-se também dificuldade em manejar os atendimentos e encaminhamentos, em função das mudanças de fluxos na atenção primária e sobrecarga do sistema devido a pandemia, pois os referenciamentos às especialidades ou mesmo outros pontos na rede de cuidados ficaram limitados devido a COVID-19. Sendo este um momento de adaptação para todos os envolvidos, inclusive a gestão dos serviços, cujo apoio é fundamental para a continuidade do cuidado na atenção primária, ${ }^{(14,17)}$ com fortalecimento da articulação entre setores e serviços para atender as demandas dos usuários e suas famílias. ${ }^{(14)}$

Como a maioria dos contatos tem sido remoto, uma preocupação evidenciada pelos enfermeiros foi a perda de vínculo com as famílias e o comprometimento do cuidado integral, essencial para o seguimento das crianças na atenção primária. Por outro lado, para não deixar o atendimento completamente de lado, o uso de alternativas remotas de acesso e vínculo com as famílias, como as redes de mensagem e telefone foram amplamente mencionados, indicando essa como uma possibilidade a ser adotada definitivamente como uma oportunidade de atenção à saúde. ${ }^{(15,17)}$

Em tempos que se é possível trabalhar a partir do uso de tecnologias digitais, os serviços de saúde não podem ficar distantes de tais transformações. ${ }^{(13)}$ Nesse cenário, o enfermeiro tem potencial para assumir papel de liderança, se destacar e interagir ainda mais com os usuários; e, com capacitação constante e por meio de uma prática interprofissional, ofertar cuidado qualificado em saúde. ${ }^{(18)} \mathrm{A}$ inclusão das ferramentas digitais no cotidiano da prática assistencial dos profissionais de saúde da APS pode perpassar o momento atual decorrente da pandemia da COVID-19 e ser empregada como rotina para, por exemplo, realizar a busca ativa ou mesmo fazer o monitoramento dos usuários com condições crônicas.

\section{Conclusão}

Esse estudo permitiu conhecer como os enfermeiros de unidades de saúde (USF e UBS) em três municípios brasileiros têm enfrentado as demandas de cuidado da criança, frente à pandemia da COVID-19. Identificando-se que nesse período, independente do tipo de unidade de saúde, o atendimento presencial foi mantido para as crianças em situações de vulnerabilidade. Assim como, o atendimento remoto por meio de novas ferramentas tecnológicas digitais passou a ser parte do cuidar da saúde da criança na APS. O reconhecimento da importância do seguimento da saúde da criança mobilizou ações para além do atendimento presencial e os sentimentos que permeiam a vivência dessa nova realidade, não somente pela equipe de saúde como também pela família, tem fortalecido a oportunidade de orientações em saúde ampliadas. O que se desvela são novos modos de pensar a assistência à criança, novas ocasiões para viabilizar cuidados que atenda às necessidades de saúde dessa população e de sua família. Considerar as mudanças, pelas quais os enfermeiros se permitiram transformar e inovar as possibilidades de cuidado da criança, durante pan- 
demia, demonstram que esforços têm sido envidados para sustentar a qualidade do cuidado infantil. O empenho dos enfermeiros e as estratégias, aqui identificadas, devem ser considerados por setores e serviços de saúde brasileiros que ainda carecem de olhar, de modo integral, para a criança e sua família. Em tempos de pandemia, faz-se premente impedir que fragilidades no atendimento tornem vulneráveis as crianças atendidas por serviços de saúde.

\section{Contribuições}

Toso BRGO, Viera CS, Furtado MCC e Bonati PCR declaram que contribuíram com a concepção do estudo, coleta, análise e interpretação dos dados, redação do artigo, revisão crítica relevante do conteúdo intelectual e aprovação da versão final a ser publicada.

\section{Agradecimentos}

As secretarias municipais de saúde dos três municípios brasileiros participantes do estudo que não mediram esforços para possibilitar a realização da coleta de dados com os enfermeiros de seu quadro de profissionais.

\section{Referências}

1. Brasil. Ministério da Saúde. Secretaria de Atenção à Saúde. Departamento de Ações Programáticas Estratégicas. Política Nacional de Atenção Integral à Saúde da Criança: orientações para implementação. Brasília (DF): Ministério da Saúde; 2018.
2. Brasil. Ministério da Saúde. Secretaria de Atenção à Saúde. Departamento de Atenção Básica. Saúde da criança: crescimento e desenvolvimento. Brasília (DF): Ministério da Saúde; 2012.

3. Coronavírus: 0 mapa que mostra 0 alcance mundial da doença [Internet]. BBC News; 2020 [atualizado 2020 Ago; citado 2020 Jul 15]. Disponível em: https://www.bbc.com/ portuguese/brasil-52472202

4. World Health Organization (WHO). Coronavirus disease 2019 (COVID-19): Situation report - 177. 2020. Genève: WHO; 2020 [updated 2020 Jan 27; cited 2020]. Available from: https://www.who.int/emergencies/diseases/novel-coronavirus-2019/situation-reports

5. Zimmermann P, Curtis N. COVID-19 in children, pregnancy and neonates: a review of epidemiologic and clinical features. Pediatr Infect Dis J. 2020;39(6):469-77.

6. Brasil. Ministério da Saúde. Secretaria de Atenção à Saúde. Departamento de Ações Programáticas Estratégicas. Orientações para manejo de pacientes com COVID-19. Brasília (DF): Ministério da Saúde; 2020.

7. Marconi MA, Lakatos EM. Técnicas de pesquisa: planejamento e execução de pesquisas, amostragens e técnicas de pesquisa, elaboração, análise e intepretação de dados. 7th ed. São Paulo: Atlas; 2011

8. Bardin L. Análise de conteúdo. São Paulo: Edições 70; 2011.

9. Ferreira SR, Périco LA, Dias VR. A complexidade do trabalho do enfermeiro na Atenção Primária à Saúde. Rev Bras Enferm. 2018;71(Supl 1):752-7.

10. Monteiro AT, Ferraria RA, Tacla MT, Souza AL. Consulta de enfermagem à criança após alta das maternidades: seguimento na atenção primária. Rev Soc Bras Enferm Ped. 2017;17(1):7-13.

11. Gaíva MA,Alves MD, Monteschio CA. Consulta de enfermagem em puericultura na estratégia saúde da família. Rev Soc Bras Enferm Ped. 2019;19(2):65-73.

12. Coronavirus disease 2019 (COVID-19). The right clinical information, right where it's needed. BMJ Best Practice; 2020 [last updated 2020 May 1; cited 2020 Sep 25]. Available from: https://bestpractice.bmj.com/topics/en-us/3000168/pdf/3000168/ Coronavirus\%20disease\%202019\%20\%28COVID-19\%29.pdf

13. Sanches JF. Telemedicina: 0 futuro que a pandemia aproximou. Gazeta Méd. 2020;1-4.

14. Fundação Oswaldo Cruz (FlOCRUZ). Saúde Mental e Atenção Psicossocial na Pandemia da COVID-19: Crianças na Pandemia COVID-19. FOCRUZ; 2020 [citado 2020 Set 23]. Disponivel em: https://www.fiocruzbrasilia.fiocruz.br/wp-content/uploads/2020/05/crianças_pandemia.pdf.

15. Sarti TD, Lazarini WS, Fontenelle LF, Almeida AP. Qual 0 papel da Atenção Primária à Saúde diante da pandemia provocada pela COVID-19? Epidemiol Serv Saude. 2020;29(2):e2020166.

16. Schmidt B, Crepaldi MA, Bolze SD, Neiva-Silva L, Demenech LM. Saúde mental e intervenções psicológicas diante da pandemia do novo coronavírus (COVID-19). Estud Psicol. 2020;37:e200063.

17. Tasca R, Massuda A. Estratégias para reorganização da Rede de Atenção à Saúde em resposta à Pandemia COVID-19: a experiência do Sistema de Saúde Italiano na região de Lazio. APS Rev. 2020;2(1):20-7.

18. Lapão LV.A Enfermagem do futuro: combinando saúde digital e a liderança do enfermeiro. Rev Lat Am Enfermagem. 2020;28:e3338. 